Revista Bioética

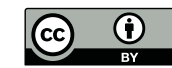

Print version ISSN 1983-8042 On-line version ISSN 1983-8034

Rev. Bioét. vol.28 no.4 Brasília Out./Dez. 2020

Doi: 10.1590/1983-80422020284434

\title{
PESQUISA
}

\section{Dilemas éticos em cuidados paliativos: revisão de literatura}

Fabíola Alves Alcântara

Universidade Federal do Rio de Janeiro, Rio de Janeiro/RJ, Brasil.

\section{Resumo}

Em qualquer sociedade, assuntos concernentes ao início e fim da vida são imenso desafio, pois envolvem diversos conceitos relacionados a ciência e religião, de caráter humanístico, social, jurídico, bioético e moral. 0 objetivo deste estudo é apresentar os dilemas éticos explicitados por profissionais de saúde ao enfrentar processos de finitude. Dentre os diversos tipos de dilemas éticos encontrados, os principais perpassam a limitação de recursos, a dificuldade de comunicação e a dificuldade em tomar decisões. Esses dilemas levam a refletir sobre uma série de cuidados necessários, como estabelecer comunicação integrada, sempre dizer a verdade e entender renúncias, descontinuações e recusas ao tratamento.

Palavras-chave: Bioética. Tomada de decisões. Assistência terminal. Pessoal de saúde.

\section{Resumen}

\section{Dilemas éticos en cuidados paliativos: revisión de la literatura}

En cualquier sociedad, las cuestiones relativas al inicio y al final de la vida constituyen un inmenso desafío, ya que involucran varios conceptos relacionados con la ciencia y la religión, de carácter humanista, social, jurídico, bioético y moral. El objetivo del estudio es presentar los dilemas éticos explicitados por profesionales de la salud cuando están frente a procesos de finitud. Entre los diversos tipos de dilemas éticos encontrados, los principales son los recursos limitados, las dificultades de comunicación y las dificultades para tomar decisiones. Estos dilemas nos llevan a reflexionar sobre una serie de cuidados que debemos emplear en esos procesos de finitud, como establecer una comunicación integrada, decir siempre la verdad y comprender las renuncias, interrupciones y negativas al tratamiento.

Palabras clave: Bioética. Toma de decisiones. Cuidado terminal. Personal de salud.

\section{Abstract}

\section{Ethical dilemmas in palliative care: a literature review}

Issues concerning the beginning and end of life are an immense challenge for they involve various concepts related to science and religion, and have humanistic, social, legal, bioethical, and moral character. This study aimed to present the ethical dilemmas experienced by health professionals when facing death. Among the various ethical impasses, the main ones are resource limitations, the difficulty in communication and decision-making. They make us reflect on various end-of-life factors, such as establishing integrated communication, always telling the truth, and understanding non-adherence, discontinuations, and treatment refusals.

Keywords: Bioethics. Decision making. Terminal care. Health personnel. 
Com seu formato peculiar de cuidado, as médicas Cicely Saunders e Elisabeth Kübler-Ross muito influenciaram o desenvolvimento dos cuidados paliativos $^{1}$. De acordo com Doyle e colaboradores ${ }^{2}$, o movimento hospice, idealizado por Saunders, contraria o tratamento então dispensado aos doentes em final de vida, cujo sofrimento se fundava na dor, sendo grande a preocupação em elaborar formas de controlar esse sintoma ${ }^{3}$. Criado na Grã-Bretanha, esse novo modelo se disseminou pelos Estados Unidos, pela França e por diversos outros países, tornando Saunders a precursora dos cuidados paliativos no mundo ${ }^{4-5}$.

A Organização Mundial da Saúde (OMS) primeiro definiu "cuidados paliativos" em 1986, mas o conceito foi ampliado de acordo com realidades e perfis epidemiológicos locais, sendo redefinido em 2002 como abordagem para melhoria da qualidade de vida de pacientes e familiares que enfrentem (...) doença ameaçadora da vida, por meio de prevenção $e$ alívio do sofrimento com identificação precoce $e$ impecável avaliação e tratamento da dor e de outros problemas físicos, psicossociais e espirituais ${ }^{6}$.

Matsumoto elencou os princípios norteadores da prática multiprofissional em cuidados paliativos preconizados pela OMS: promover alívio da dor $e$ outros sintomas desagradáveis; afirmar a vida e considerar a morte como um processo normal da vida; não acelerar nem adiar a morte; integrar os aspectos psicológicos e espirituais no cuidado ao paciente; oferecer um sistema de suporte que possibilite ao paciente viver tão ativamente quanto possível, até o momento da sua morte; oferecer sistema de suporte para auxiliar os familiares durante a doença do paciente e a enfrentar o luto; abordagem multiprofissional para focar as necessidades dos pacientes e seus familiares, incluindo acompanhamento no luto; melhorar a qualidade de vida e influenciar positivamente o curso da doença; deve ser iniciado o mais precocemente possivel e incluir todas as investigações necessárias para melhor compreender e controlar situações clínicas estressantes ${ }^{7}$.

A assistência nos cuidados paliativos precisa ser integral, abrangendo diversos segmentos, entre eles o físico, espiritual, psicológico e social, envolvendo olhar cuidadoso e equipe multidisciplinar ${ }^{8}$. $O$ enfoque multiprofissional permite à equipe trocar conhecimentos e facilita o entendimento das fragilidades e condições do enfermo sem possibilidade de cura $^{9,10}$. Esse enfoque tem grande importância para os cuidados paliativos porque demonstra o quão fundamental é cada profissão, que individualmente não consegue abarcar todas as nuances do atendimento a esses pacientes, destacando o papel da coletividade para a adequada assistência ${ }^{9,11}$.
Assuntos concernentes ao início e fim da vida são imenso desafio em qualquer sociedade, pois envolvem conceitos relacionados a ciência e religião, de caráter humanístico, social, jurídico, bioético e moral $^{12,13}$. As diferenças culturais levam cada nação a abordar a questão de modo diverso, e Vasconcelos e Pereira ${ }^{14}$ apontam que Inglaterra, Austrália e Nova Zelândia são os países que mais investem em cuidados paliativos, conforme relatório sobre qualidade de morte de 2015. Concentrando-se nas implicações do fim da vida, as decisões tomadas sobre o tratamento e o processo de morte são relevantes. Tais dilemas éticos demandam reflexões que envolvem toda a sociedade acerca das condutas mais apropriadas ${ }^{15,16}$.

Para Medeiros e colaboradores, o dilema ético está permeado pela cultura, filosofia e pelos valores que [as] baseiam (...). A pluralidade dos diferentes contextos culturais de uma sociedade que são constituídas (sic) de indivíduos que hierarquizam seus valores de maneira singular baseados pela autonomia permite constantes indagações sobre as questões que envolvem a vida e, principalmente, sua terminalidade ${ }^{17}$.

Os dilemas éticos carregam consigo duas ou mais opções terapêuticas ou direcionamentos adequados a determinada situação, permeando a prática dos profissionais da saúde, a qual é suscetível à indagação moral ou social ${ }^{18,19}$. A temática dos dilemas traz diversas preocupações, motivando a coletividade científica a estudá-la incessantemente nos eixos legal e ético ${ }^{20}$. Com isso, este trabalho objetiva identificar na literatura os dilemas éticos vivenciados por profissionais de saúde no atendimento a pacientes sem possibilidades terapêuticas de cura.

\section{Método}

Trata-se de revisão de literatura incluindo artigos cujo texto completo está disponível online. Foi realizada busca nas bases de dados Medical Literature Analysis and Retrieval System Online, Biblioteca Regional de Medicina, Scientific Electronic Library Online e Literatura Latino-Americana e do Caribe em Ciências da Saúde. Utilizaram-se descritores em português e seus correspondentes em inglês e espanhol: "bioética", "bioethics", "bioética"; "tomada de decisões", "decision making", "toma de decisiones"; "assistência terminal", "terminal care", "cuidado terminal"; e "pessoal de saúde", "health personnel", "personal de salud". Foram excluídos relatos de caso e trabalhos que, apesar de apresentarem os descritores elencados, referiam dilemas éticos vivenciados 
por pacientes ou seus familiares. Foram identificados 51 artigos sobre o tema e, depois de empregados os critérios, obteve-se amostra de 14 artigos.

Fontes e descritores foram selecionados visando enfocar artigos que referiam os dilemas éticos vivenciados por profissionais de saúde no atendimento a pacientes em fim de vida, sem delimitar seu ano de publicação. Para viabilizar a análise, a amostra foi classificada quanto a autoria, ano de publicação, título do artigo, objetivo, método e nome do periódico em que foi publicado. O Quadro 1 apresenta os artigos selecionados.

Quadro 1. Corpus da revisão não sistemática da literatura

\begin{tabular}{|c|c|c|c|c|}
\hline Autor(es)/ano & Título & Periódico & Objetivo & Método \\
\hline $\begin{array}{l}\text { Araújo, Neves; } \\
2003^{21}\end{array}$ & $\begin{array}{l}\text { "A bioética e a fisioterapia } \\
\text { nas unidades de terapia } \\
\text { intensiva" }\end{array}$ & $\begin{array}{l}\text { Revista de } \\
\text { Fisioterapia da } \\
\text { Universidade } \\
\text { de São Paulo }\end{array}$ & $\begin{array}{l}\text { Traçar o perfil dos profissionais da } \\
\text { fisioterapia que trabalham em unidades } \\
\text { de terapia intensiva e identificar sua } \\
\text { opinião acerca de temas como pacientes } \\
\text { terminais, autonomia do paciente, } \\
\text { eutanásia e distanásia. }\end{array}$ & $\begin{array}{l}\text { Pesquisa de } \\
\text { campo }\end{array}$ \\
\hline Barnitt; $1998^{22}$ & $\begin{array}{l}\text { "Ethical dilemmas in } \\
\text { occupational therapy and } \\
\text { physical therapy: a survey } \\
\text { of practitioners in the UK } \\
\text { National Health Service" }\end{array}$ & $\begin{array}{c}\text { Journal of } \\
\text { Medical Ethics }\end{array}$ & $\begin{array}{l}\text { Identificar os dilemas éticos } \\
\text { experimentados por terapeutas } \\
\text { ocupacionais e fisioterapeutas que } \\
\text { trabalham no Serviço Nacional de Saúde do } \\
\text { Reino Unido e comparar contextos éticos, } \\
\text { temas e princípios entre os dois grupos. }\end{array}$ & $\begin{array}{c}\text { Pesquisa de } \\
\text { campo }\end{array}$ \\
\hline $\begin{array}{l}\text { Bélanger, } \\
\text { Rodríguez, } \\
\text { Groleau; } \\
2011^{23}\end{array}$ & $\begin{array}{l}\text { "Shared decision-making in } \\
\text { palliative care: a systematic } \\
\text { mixed studies review using } \\
\text { narrative synthesis" }\end{array}$ & $\begin{array}{l}\text { Palliative } \\
\text { Medicine }\end{array}$ & $\begin{array}{l}\text { Sintetizar o conhecimento sobre } \\
\text { o processo de tomada de decisão } \\
\text { compartilhada em cuidados paliativos. }\end{array}$ & $\begin{array}{l}\text { Revisão de } \\
\text { literatura }\end{array}$ \\
\hline $\begin{array}{l}\text { Chih e } \\
\text { colaboradores; } \\
2016^{24}\end{array}$ & $\begin{array}{l}\text { "The changes of ethical } \\
\text { dilemmas in palliative } \\
\text { care: a lesson learned from } \\
\text { comparison between } 1998 \\
\text { and } 2013 \text { in Taiwan" }\end{array}$ & $\begin{array}{l}\text { Medicine } \\
\text { (Baltimore) }\end{array}$ & $\begin{array}{l}\text { Investigar os dilemas éticos } \\
\text { encontrados por médicos e } \\
\text { enfermeiros de cuidados paliativos em } \\
2013 \text { e comparar os resultados com } \\
\text { pesquisa realizada em } 1998 .\end{array}$ & $\begin{array}{l}\text { Pesquisa de } \\
\text { campo }\end{array}$ \\
\hline $\begin{array}{l}\text { Gjerberg e } \\
\text { colaboradores; } \\
2010^{25}\end{array}$ & $\begin{array}{l}\text { "Ethical challenges in the } \\
\text { provision of end-of-life } \\
\text { care in Norwegian nursing } \\
\text { homes" }\end{array}$ & $\begin{array}{l}\text { Social Science } \\
\text { and Medicine }\end{array}$ & $\begin{array}{l}\text { Examinar os tipos e a prevalência dos } \\
\text { desafios éticos nos cuidados de } \\
\text { fim de vida. }\end{array}$ & $\begin{array}{l}\text { Pesquisa de } \\
\text { campo }\end{array}$ \\
\hline $\begin{array}{l}\text { Mobasher e } \\
\text { colaboradores; } \\
2013^{26}\end{array}$ & $\begin{array}{l}\text { "Ethical issues in the end } \\
\text { of life care for cancer } \\
\text { patients in Iran" }\end{array}$ & $\begin{array}{l}\text { Iranian Journal } \\
\text { of Public } \\
\text { Health }\end{array}$ & $\begin{array}{l}\text { Identificar os dilemas éticos que os } \\
\text { oncologistas iranianos podem enfrentar } \\
\text { no ambiente de saúde e determinar os } \\
\text { fatores que influenciam o processo de } \\
\text { tomada de decisão. }\end{array}$ & $\begin{array}{l}\text { Pesquisa de } \\
\text { campo }\end{array}$ \\
\hline $\begin{array}{l}\text { Sorta-Bilajac e } \\
\text { colaboradores; } \\
2011^{27}\end{array}$ & $\begin{array}{l}\text { "How nurses and } \\
\text { physicians face ethical } \\
\text { dilemmas: the Croatian } \\
\text { experience" }\end{array}$ & Nursing Ethics & $\begin{array}{l}\text { Avaliar os dilemas éticos de enfermeiros } \\
\text { e médicos na prática clínica. }\end{array}$ & $\begin{array}{l}\text { Pesquisa de } \\
\text { campo }\end{array}$ \\
\hline $\begin{array}{l}\text { Granero- } \\
\text { Moya e } \\
\text { colaboradores; } \\
2016^{28}\end{array}$ & $\begin{array}{l}\text { "Dificultades de las } \\
\text { enfermeras de atención } \\
\text { primaria en los procesos de } \\
\text { planificación anticipada de } \\
\text { las decisiones: un estudio } \\
\text { cualitativo" }\end{array}$ & $\begin{array}{l}\text { Atención } \\
\text { Primaria }\end{array}$ & $\begin{array}{l}\text { Conhecer as dificuldades encontradas } \\
\text { por enfermeiras de atenção primária } \\
\text { para estabelecer as diretivas antecipadas } \\
\text { de vontade de pessoas em estado } \\
\text { terminal. }\end{array}$ & $\begin{array}{l}\text { Pesquisa de } \\
\text { campo }\end{array}$ \\
\hline $\begin{array}{l}\text { Guardia } \\
\text { Mancilla e } \\
\text { colaboradores; } \\
2018^{29}\end{array}$ & $\begin{array}{l}\text { "Percepciones de los } \\
\text { profesionales sobre } \\
\text { la atención prestada, } \\
\text { obstáculos y dilemas éticos } \\
\text { relacionados con el final de } \\
\text { la vida en hospitales, centros } \\
\text { de atención primaria y } \\
\text { residencias de ancianos" }\end{array}$ & $\begin{array}{l}\text { Anales del } \\
\text { Sistema } \\
\text { Sanitario de } \\
\text { Navarra }\end{array}$ & $\begin{array}{l}\text { Comparar a percepção da prática } \\
\text { profissional e os dilemas éticos de } \\
\text { médicos e enfermeiros na assistência } \\
\text { terminal na atenção primária, hospitalar } \\
\text { e em residências de idosos. }\end{array}$ & $\begin{array}{c}\text { Estudo } \\
\text { descritivo, } \\
\text { transversal e } \\
\text { multicêntrico }\end{array}$ \\
\hline
\end{tabular}


Quadro 1. Continuação

\begin{tabular}{|c|c|c|c|c|}
\hline Autor(es)/ano & Título & Periódico & Objetivo & Método \\
\hline $\begin{array}{l}\text { Sánchez- } \\
\text { García e } \\
\text { colaboradores; } \\
2017^{30}\end{array}$ & $\begin{array}{l}\text { "Dificultades y factores } \\
\text { favorables para la atención } \\
\text { al final de la vida en } \\
\text { residencias de ancianos: } \\
\text { un estudio con grupos } \\
\text { focales" }\end{array}$ & $\begin{array}{l}\text { Atención } \\
\text { Primaria }\end{array}$ & $\begin{array}{l}\text { Identificar dificuldades e fatores } \\
\text { enfrentados por profissionais que atuam } \\
\text { em residências de idosos. }\end{array}$ & $\begin{array}{l}\text { Pesquisa de } \\
\text { campo }\end{array}$ \\
\hline $\begin{array}{l}\text { Motta e } \\
\text { colaboradores; } \\
2016^{31}\end{array}$ & $\begin{array}{l}\text { "Tomada de decisão } \\
\text { em (bio)ética } \\
\text { clínica: abordagens } \\
\text { contemporâneas" }\end{array}$ & $\begin{array}{l}\text { Revista } \\
\text { Bioética }\end{array}$ & $\begin{array}{l}\text { Identificar situações que angustiam } \\
\text { profissionais de saúde e/ou pesquisadores } \\
\text { na prática clínica e apresentar } \\
\text { sucintamente reflexões ou abordagens } \\
\text { correlacionadas ao processo decisório. }\end{array}$ & $\begin{array}{l}\text { Revisão de } \\
\text { literatura }\end{array}$ \\
\hline $\begin{array}{l}\text { Renner, } \\
\text { Goldim, Prati; } \\
2002^{18}\end{array}$ & $\begin{array}{l}\text { "Dilemas éticos } \\
\text { presentes na prática do } \\
\text { fisioterapeuta" }\end{array}$ & $\begin{array}{c}\text { Revista } \\
\text { Brasileira de } \\
\text { Fisioterapia }\end{array}$ & $\begin{array}{l}\text { Identificar tipos de dilemas éticos } \\
\text { presentes na prática do fisioterapeuta. }\end{array}$ & $\begin{array}{l}\text { Pesquisa de } \\
\text { campo }\end{array}$ \\
\hline $\begin{array}{l}\text { Pereira; } \\
2007^{32}\end{array}$ & $\begin{array}{l}\text { "Dilemas éticos ligados à } \\
\text { prática do fisioterapeuta } \\
\text { no atendimento da criança } \\
\text { com deficiência física ou } \\
\text { mental" }\end{array}$ & $\begin{array}{l}\text { Dissertação de } \\
\text { mestrado }\end{array}$ & $\begin{array}{l}\text { Verificar na prática profissional de } \\
\text { fisioterapeutas os dilemas éticos que } \\
\text { orientam sua relação terapêutica com } \\
\text { a criança com deficiência física e/ou } \\
\text { mental e sua família. }\end{array}$ & $\begin{array}{l}\text { Pesquisa de } \\
\text { campo }\end{array}$ \\
\hline $\begin{array}{l}\text { Lorenzo, } \\
\text { Bueno; } 2013^{33}\end{array}$ & $\begin{array}{l}\text { "A interface entre bioética } \\
\text { e fisioterapia nos artigos } \\
\text { brasileiros indexados" }\end{array}$ & $\begin{array}{l}\text { Fisioterapia } \\
\text { em } \\
\text { Movimento }\end{array}$ & $\begin{array}{l}\text { Estudar as temáticas e a fundamentação } \\
\text { teórica dos artigos indexados que } \\
\text { pretendiam discutir a interface entre } \\
\text { fisioterapia e bioética. }\end{array}$ & $\begin{array}{l}\text { Revisão de } \\
\text { literatura }\end{array}$ \\
\hline
\end{tabular}

\section{Resultados e discussão}

Gjerberg e colaboradores ${ }^{25}$ buscaram identificar os tipos de desafios éticos e sua prevalência na visão de 664 profissionais de saúde que atuavam em enfermarias de lares de idosos da Noruega. Os entrevistados apontaram com maior frequência a inadequação do cuidado pela escassez de recursos e o desrespeito à autonomia e integridade do indivíduo ${ }^{25}$. Essas observações corroboram os resultados encontrados por Lorenzo e Bueno ${ }^{33}$, que descrevem vários conflitos na prática profissional de fisioterapeutas, com destaque para os relacionados a início e fim de vida, autonomia do sujeito, recusa de tratamento e tomada de decisão quando a autonomia é de alguma forma limitada.

Guardia Mancilla e colaboradores ${ }^{29}$ aplicaram questionário a 378 profissionais espanhóis que atuavam em hospitais, atenção primária e asilos, verificando que os dilemas éticos mais frequentes relacionados ao fim de vida estavam voltados ao uso das diretivas antecipadas de vontade. Granero-Moya e colaboradore ${ }^{28}$ investigaram as dificuldades enfrentadas por enfermeiros na atenção primária em processos de tomada de decisão em casos terminais. Constataram pouco conhecimento sobre o assunto, dificuldades comunicativas, falta de tempo e relação interprofissional deficiente ${ }^{28}$.
Em estudo com oncologistas iranianos, Mobasher e colaboradores ${ }^{26}$ perceberam como dilemas éticos dizer a verdade, a definição incerta do quadro do enfermo e a relação equipe-paciente. Esses achados reforçam o estudo de Motta e colaboradores ${ }^{31}$, que detectaram as inquietudes dos profissionais de saúde na atuação clínica e a dificuldade enfrentada no processo de tomada de decisão, em que casos de origem e finitude de vida causam mais aflição e atritos. Observa-se que dois aspectos da clínica muito difíceis para profissionais e equipes de saúde são a comunicação de más notícias e o momento de estabelecer cuidados paliativos ${ }^{31}$.

Sánchez-García e colaboradores ${ }^{30}$ identificaram problemas na comunicação da equipe com as famílias e falta de incentivo à participação dos doentes na tomada de decisão como as principais dificuldades vivenciadas por profissionais na atenção a idosos que moravam em casas de repouso espanholas. Já para Sorta-Bilajac e colaboradores ${ }^{27}$, os dilemas éticos enfrentados por médicos e enfermeiros croatas em sua prática clínica estão voltados a eutanásia, suicídio assistido e limitação de terapias para manutenção da vida. Em estudo comparativo, Chih e colaboradores ${ }^{24}$ descreveram as mudanças nos dilemas éticos em cuidados paliativos entre 1998 e 2013 em Taiwan. Em 1998 observaram-se dificuldade em orientar os enfermos, famílias que não queriam levar o paciente para 
casa e omissão da verdade por parte da família aos doentes, enquanto em 2013 os dilemas éticos estavam relacionados à alocação de recursos.

De acordo com Barnitt ${ }^{22}$, os dilemas éticos com os quais se deparam fisioterapeutas em sua prática ainda são pouco conhecidos. Em seu estudo com esses profissionais do Serviço Nacional de Saúde da Inglaterra, os resultados apontaram alocação injusta e falta de recursos, baixa eficácia do tratamento fisioterapêutico, decisões terapêuticas divergentes entre a equipe, desrespeito à confidencialidade e autonomia do paciente e omissão da verdade. A ocorrência de dilemas éticos prevaleceu, segundo participantes da pesquisa, no ambiente hospitalar ${ }^{22}$.

Renner, Goldim e Prati ${ }^{18}$, por sua vez, identificaram, por meio de questionário, que a maioria dos dilemas relatados por fisioterapeutas atuantes em Porto Alegre/RS se baseava no limite de atuação profissional, seguido por omissão da verdade e falta de recursos e eficiência da terapia, chamando atenção o considerável número de questionários que acusavam ausência de dilemas. O resultado corrobora o estudo de Pereira ${ }^{32}$, que investigou a mesma categoria profissional, mas no atendimento a crianças com deficiência física e/ou mental e familiares na cidade de Campinas/SP. A autora concluiu que muitos dos respondentes, a maioria com menos de 15 anos de profissão, estão preocupados e comprometidos com a ética, mas não identificaram dilemas em sua atuação profissional, e sim questões cotidianas, como as relacionadas com a alta fisioterapêutica ${ }^{32}$.

\section{Considerações finais}

Os dilemas éticos enfrentados em situações de terminalidade por profissionais de saúde levam a refletir sobre diversos cuidados necessários ao processo de morte. Exemplos desses cuidados são a comunicação integrada entre profissional, paciente e familiares, o dever de informar sempre a verdade e a necessidade de compreender renúncias, descontinuações e recusa de tratamentos.

O processo de tomada de decisão vem sendo um dos principais obstáculos para os profissionais de saúde em assistência terminal, destacando-se a insegurança, a falta de preparo profissional e a carência de conhecimento sobre a temática discutida. Vale ressaltar a importância de realizar outros estudos com a intenção de avaliar o que esses profissionais ponderam no processo de tomada de decisão quando se deparam com algum desses dilemas.

\section{Referências}

1. Menezes RA. Em busca da boa morte: antropologia dos cuidados paliativos. Rio de Janeiro: Garamond; 2004.

2. Doyle D, Hanks G, Cherny N, Calman K. Introduction. In: Doyle D, Hanks G, Cherny N, Calman K, editores. Oxford textbook of palliative medicine. 3a ed. New York: Oxford University Press; 2003. p. 1.

3. Clark D, Seymour J. Reflections on palliative care. Buckingham: Open University Press; 1999.

4. Marinho SO. Cuidados paliativos e práticas de saúde: um estudo sobre a gestão da morte na sociedade contemporânea [tese] [Internet]. Rio de Janeiro: Universidade do Estado do Rio de Janeiro; 2010 [acesso 13 fev 2019]. Disponível: https://bit.ly/3jChMam

5. Pessini L. Cuidados paliativos: alguns aspectos conceituais, biográficos e éticos. Prat Hosp. 2005;(41):107-12.

6. Palliative care. World Health Organization [Internet]. c2020 [acesso 2 dez 2020]. Tradução livre. Disponível: https://bit.ly/3jD6zGi

7. Matsumoto DY. Cuidados paliativos: conceito, fundamentos e princípios. In: Carvalho RT, Parsons HA organizadores. Manual de cuidados paliativos ANCP. 2a ed. Rio de Janeiro: ANCP; 2012. p. 23-30. p. 26.

8. Hermes HR, Lamarca ICA. Cuidados paliativos: uma abordagem a partir das categorias profissionais de saúde. Ciênc Saúde Coletiva [Internet]. 2013 [acesso 24 abr 2019];18(9):2577-88. DOI: 10.1590/ S1413-81232013000900012

9. Reis LC Jr, Reis PEAM. Cuidados paliativos no paciente idoso: o papel do fisioterapeuta no contexto multidisciplinar. Fisioter Mov [Internet]. 2007 [acesso 24 abr 2019];20(2):127-35. Disponível: https://bit.ly/33CbDoS

10. Girão M, Alves S. Fisioterapia nos cuidados paliativos. Salutis Scientia [Internet]. 2013 [acesso 24 abr 2019];5:34-41. Disponível: https://bit.ly/3nk672d

11. McCoughlan MA. Necessidade de cuidados paliativos. O Mundo da Saúde. 2003;27(1):6-14.

12. Almeida M. Considerações de ordem ética sobre o início e o fim da vida [tese]. São Paulo: Faculdade de Medicina da Universidade de São Paulo; 1988.

13. Medeiros MB, Pereira ER, Silva RMCRA, Silva MA. Dilemas éticos em UTI: contribuições da teoria dos valores de Max Scheler. Rev Bras Enferm [Internet]. 2012 [acesso 20 jul 2020];65(2):276-84. DOI: 10.1590/S0034-71672012000200012 
14. Vasconcelos GB, Pereira PM. Cuidados paliativos em atenção domiciliar: uma revisão bibliográfica. Rev Adm Saúde [Internet]. 2018 [acesso 24 abr 2019];18(70). DOI: 10.23973/ras.70.85

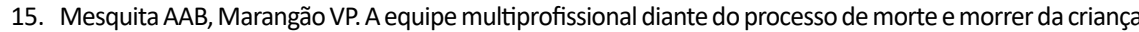
hospitalizada [monografia]. Campos Gerais: Faculdade de Ciências da Saúde de Campos Gerais; 2008.

16. Paiva FCL, Almeida JJ Jr, Damasio AC. Ética em cuidados paliativos: concepções sobre o fim da vida. Rev. bioét. (Impr.) [Internet]. 2014 [acesso 24 abr 2019];22(3):550-60. DOI: 10.1590/1983-80422014223038

17. Medeiros MB, Pereira ER, Silva RMCRA, Silva MA. Op. cit. p. 277.

18. Renner AF, Goldim JR, Prati FM. Dilemas éticos presentes na prática do fisioterapeuta. Braz J Phys Ther. 2002;6(3):135-8.

19. Chaves AAB, Massarollo MCKB. Percepção de enfermeiros sobre dilemas éticos relacionados a pacientes terminais em unidades de terapia intensiva. Rev Esc Enferm USP [Internet]. 2009 [acesso 24 abr 2019];43(1):30-6. DOI: 10.1590/S0080-62342009000100004

20. Junges JR, Cremonese C, Oliveira EA, Souza LL, Backes V. Reflexões legais e éticas sobre o final da vida: uma discussão sobre a ortotanásia. Rev. bioét. (Impr.) [Internet]. 2010 [acesso 24 abr 2019];18(2):275-88. Disponivel: https://bit.ly/3nwwnGL

21. Araújo LZS, Neves WA Jr. A bioética e a fisioterapia nas unidades de terapia intensiva. Rev Fisioter Univ São Paulo [Internet]. 2003 [acesso 18 ago 2018];10(2):52-60. Disponível: https://bit.ly/30FOkbN

22. Barnitt R. Ethical dilemmas in occupational therapy and physical therapy: a survey of practitioners in the UK National Health Service. J Med Ethics [Internet]. 1998 [acesso 22 out 2020];24(3):193-9. DOI: 10.1136/jme.24.3.193

23. Bélanger $E$, Rodríguez $C$, Groleau D. Shared decision-making in palliative care: a systematic mixed studies review using narrative synthesis. Palliat Med [Internet]. 2011 [acesso 10 jan 2019];25(3):242-61. DOI: 10.1177/0269216310389348

24. Chih AH, Su P, Hu WY, Yao CA, Cheng SY, Lin YC, Chiu TY. The changes of ethical dilemmas in palliative care: a lesson learned from comparison between 1998 and 2013 in Taiwan. Medicine [Internet]. 2016 [acesso 24 abr 2019];95(1):e2323. DOI: 10.1097/MD.0000000000002323

25. Gjerberg E, Førde R, Pedersen R, Bollig G. Ethical challenges in the provision of end-of-life care in Norwegian nursing homes. Soc Sci Med [Internet]. 2010 [acesso 24 abr 2019];71(4):677-84. DOI: 10.1016/j.socscimed.2010.03.059

26. Mobasher M, Nakhaee N, Tahmasebi M, Zahedi F, Larijani B. Ethical issues in the end of life care for cancer patients in Iran. Iran J Public Health [Internet]. 2013 [acesso 24 abr 2019];42(2):188-96. Disponível: https://bit.ly/3d90AGP

27. Sorta-Bilajac I, Baždarić K, Žagrović MB, Jančić E, Brozović B, Čengić T et al. How nurses and physicians face ethical dilemmas: the Croatian experience. Nurs Ethics [Internet]. 2011 [acesso 24 abr 2019];18(3):341-55. DOI: 10.1177/0969733011398095

28. Granero-Moya N, Frías-Osuna A, Barrio-Cantalejo IM, Ramos-Morcillo AJ. Dificultades de las enfermeras de atención primaria en los procesos de planificación anticipada de las decisiones: un estudio cualitativo. Aten Primaria [Internet]. 2016 [acesso 24 abr 2019];48(10):649-56. DOI: 10.1016/j.aprim.2016.01.008

29. Guardia Mancilla P, Montoya-Juarez R, Marti-Garcia C, Herrero Hahn R, García Caro MP, Cruz Quintana F. Percepciones de los profesionales sobre la atención prestada, obstáculos y dilemas éticos relacionados con el final de la vida en hospitales, centros de atención primaria y residencias de ancianos. An Sist Sanit Navar [Internet]. 2018 [acesso 24 abr 2019];41(1):35-46. DOI: 10.23938/ASSN.0170

30. Sánchez-García MR, Moreno-Rodríguez M, Hueso-Montoro C, Campos-Calderón C, VarellaSafont A, Montoya-Juárez R. Dificultades y factores favorables para la atención al final de la vida en residencias de ancianos: un estudio con grupos focales. Aten Primaria [Internet]. 2017 [acesso 24 abr 2019];49(5):278-85. DOI: 10.1016/j.aprim.2016.09.004

31. Motta LCS, Oliveira LN, Silva E, Siqueira-Batista R. Tomada de decisão em (bio)ética clínica: abordagens contemporâneas. Rev. bioét. (Impr.) [Internet]. 2016 [acesso 24 abr 2019];24(2):304-14. DOI: $10.1590 / 1983-80422016242131$

32. Pereira RAB. Dilemas éticos ligados à prática do fisioterapeuta no atendimento da criança com deficiência física ou mental [dissertação] [Internet]. São Paulo: Universidade Presbiteriana Mackenzie; 2007 [acesso 6 out 2020]. Disponível: https://bit.ly/3d4m0oA

33. Lorenzo CFG, Bueno GTA. A interface entre bioética e fisioterapia nos artigos brasileiros indexados. Fisioter Mov [Internet]. 2013 [acesso 24 abr 2019];26(4):763-75. DOI: 10.1590/ S0103-51502013000400006

Correspondência

Av. Franklin de Campos Sobral, 1.580 CEP 49027-000. Aracaju/SE, Brasil.

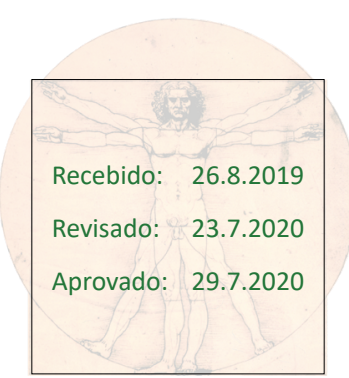

